\title{
Enterprise Computer Network Reliability Analysis
}

\author{
Yongfeng Cui, Wei Liu and Ya Li \\ School of Science and Technology, Zhoukou Normal University, Zhoukou Henan \\ 466001, China \\ cuiyf@zknu.edu.cn
}

\begin{abstract}
This paper considers the multi-protocol label switching enterprise computer network routing optimization problem, in the network reliability constraints, by selecting the optimal work and alternate paths, and carries on the bandwidth allocation; network total construction cost is minimal. Due to the total construction cost and network reliability is related to choice of the length of the path, the optimal route network design problem can be decoupled into two sub-problems: cost and reliability related issues, among them, the cost of related sub problem study, Sohn et al. For reliability related problems, reduce the search space understanding, this chapter will work best and standby path search space from a hyper plane reduce to the hyper plane line; Then, reliability related algorithm is proposed to solve the problem. Because of the cost and reliability related sub-problem solutions are obtained based on the same search criterion, so the original problem of optimal work and backup path of solution set is by comparing the two sub problems solution to work out. Finally, related algorithm provides a numerical example to verify the reliability and the validity of the global network routing design method.
\end{abstract}

Keywords: Enterprise computer network, reliability, cost of related network, search space

\section{Introduction}

With the rapid development of science and technology, the computer's widespread popularization, the computer network has been in the communications, banking, transportation, logistics, energy, schools, factories and military and other fields is widely used, in real life, everywhere the application of computer network [1]. As the user the use of computer network, people has higher requirement for the reliability of the network. Once the malfunction of the computer network will be on people's lives, economic, environmental, health, and social security have significant even disastrous effect according to the statistics, for the financial sector [2-3], if the computer network harmony risk for an hour, will cause the loss of about $\$ 6$ million, if a computer network paralysis, a day is likely to lead to an investment of commercial Banks have failed. Fault abound in real life, the computer network, related cases are: Taiwan strait earthquake in various international submarine communication optical cable is damaged, make the Internet of services in the Asia Pacific region is almost paralyzed, the Chinese mainland to Taiwan, the United States, Europe and other the direction of the communication line is affected by this is also a lot of disruption. Twitter service failure again and again, leading to network interruption, make a lot of users give up Twitter service, run to rivals such as Friend feed, resulting in a decrease of a large number of users; Amazon working days off most of the time in the United States, makes millions of users are forced to go back to work, and the expected loss of $\$ 16000$ per minute; Google online Office application software interrupt service, let the user's file to stay in the cloud, because the company established online applications such as Google is considered to replace 
the traditional applications such as Microsoft Office, the service interruption has shaken the confidence of the users for this service, resulting in loss of trust. These computer network failure events and caused huge economic loss, or caused the inconvenient for people to communicate, or cause the user as well as the mistrust of network reliability [4-6]. Therefore, enterprise network reliability research is of great significance and application value.

Study of the computer network reliability problems, as can be traced back to Mr. Lee, exploration of telecommunications switching network, due to network components failure makes telecommunications switching network transmission capacity always dropped substantially, cause call congestion, prompted a large telecommunications switching network paralysis, cause huge economic loss when Mr. Lee will call congestion as network link failures, and for the first time put forward on the basis of the connectivity of network reliability measure. Early computer network reliability research focuses in the field of communication networks. As the United States department of defense advanced research projects agency network ARPANET in computer input, the computer network reliability research obtained the huge development, caused the attention of many scholars. This time, the computer network reliability research mainly based on network connectivity as a standard for network reliability measure of network connectivity is defined as: "as long as the computer network link is connected, or as long as the need for communication between users of computer network is connected, or computer network node trouble-free, computer network is the normal work of" proves that the network connectivity probability calculation problem is NP problem, has been widely used, the network running performance effect on the reliability were studied. With the rapid development of computer technology, network scale and the rapid growth of the number of users, the network traffic and load increase sharply, network administrators found even network connectivity good can't guarantee the reliable work of computer network, network congestion and delay will lead to network performance degradation, and could lead to part of the normal operation of the business. During this period, the reliability of the network study from the connectivity of turning to for research on the reliability of the network performance. With the speeding up of the computer network and popularization, network system reliability gradually become a hot research topic in the field of reliability. At the same time, the electric power network, network circuit, the transportation network, transport network, logistics network involves computer network reliability evaluation problems, such as computer network reliability research is more and more attention [7-8].

Products under specified conditions, within a given time ability to perform the required functions [9]. The probability of reliability degree is called reliability. Accordingly, reliability is defined as: the product in a given period of time, complete the required function under given conditions and not in the probability of failure. The calculation of reliability of network by Ball scholars such as proof of NP problem, it is hard to find a general calculation method of the reliability. The current calculation method about network reliability can be divided into two categories: precision algorithm and approximate algorithm. Approximation algorithm for network reliability is a precision at the expense of reliability and reduce calculation difficulty. Common network reliability of the approximate algorithm has a lower bound on the method, the graph transformation method, simulation method, etc. With the wide application of computer network in real life, the network reliability as the focus of the managers and users concern. Due to network reliability is an important index to measure the reliability of the network, the network reliability assessment received extensive attention of the researchers. Most network reliability evaluation research is based on the premise of data 
transmitted through disjoint paths, however, in real life, the data would inevitably contain the same link through path for transmission, therefore, this paper studies the data transmitted through cross path problem of reliability assessment, the reliability assessment of network model is more realistic, have important practical research value. After the network reliability evaluation problem is studied, the next consideration based on the reliability of the computer network design problem.

\section{Related Works}

To solve the NP problem is due to network reliability, accurate calculation method is suitable for small, small and medium-sized only commonly or with special topology network. So far, the common network reliability calculation method is: state enumeration method, the principle of a class method, don't pay the product and the method, factorization method, state space decomposition method, etc.

\section{State Enumeration Method}

State enumeration method to solve the network reliability by enumeration is the major concept of the network can run normally under prescribed conditions of all mutually exclusive events to calculate reliability of network reliability can be determined by the type (1):

$$
\operatorname{Re}=\sum_{i=1}^{m} \operatorname{Pr}\left\{B_{i}\right\}
$$

All conform to the network transmission limit under the condition of network state enumeration to calculate network reliability. For binary network, if the network consists of $\mathrm{n}$ of the link, the network has kind of state $2^{n}$, using the enumeration method to calculate the complexity $o\left(2^{n}\right)$ of the network reliability. For the large network, along with the increase of the number of network link network exponentially increase in the number state, therefore, through the enumeration of all network status, it is difficult to solve the network reliability.

\section{The Principle of a Class Method}

Class theory method to calculate the network reliability is the basic principle of the network reliability said to all the path set and (or will not say to all of the minimum cut sets and reliability), and then the calculation theory of a class to get rid of incompatible event intersection parts, so as to solve the network reliability. Among them, the shortest path (minimum cut sets) is a collection of links, and the collection of any a link removed from the collection, the rest of the collection is no longer the path set (minimum cut sets).

A set of shortest path (minimum cut sets) a normal working state of the corresponding network (fault state), therefore, to maintain the normal work of the network, the network must be kept in a set or a minimum cut set the path of link failure. Assumption $A_{1}, A_{2}, \cdots, A_{m}$ is $\mathrm{m}$ events, according to the principle of a class, in the probability of at least one time for

$$
\operatorname{Pr}\left(A_{1} \cup A_{2} \cup \cdots \cup A_{m}\right)=\sum_{1 \leq i \leq m} \operatorname{Pr}\left(A_{i}\right)-\sum_{1 \leq i<j \leq m} \operatorname{Pr}\left(A_{i} A_{j}\right)+\cdots+(-1)^{m-1} \operatorname{Pr}\left(A_{1}, A_{2}, \cdots, A_{m}\right)
$$

3. Don't pay the product and the method

Don't pay the product and method (is a kind of product and theorem are used to calculate the network reliability of the method, its main idea is to network reliability, 
said to all the path set and (or will not say to all of the minimum cut sets and reliability), then this item into do not intersect each other and, thus the network reliability is calculated.

According to don't pay the product and the method to calculate the network reliability of train of thought, if the formula is not the number of the product and the less, the less computing time, and the calculation of error is smaller, therefore, people usually don't pay the product and the number of items to measure don't pay the product and the effectiveness of the algorithm. Use don't pay the product and the method to calculate the network reliability got the attention of many scholars, for the continuous improvement. With minimum cut sets based on shortest path set for a class on the same principle method to calculate the network reliability, don't pay the product and the method to calculate the network reliability also need to find out all the shortest path in the network in advance (minimum cut sets).

\section{The Factorization Method}

Using the factorization method to calculate the network reliability is the earliest by f. Markowitz, then, many scholars of the factorization method for network reliability are studied, among them, the most studied the calculation of the reliability factor decomposition method is based on the network link is based on the assumption of unreliable nodes and reliable. Factorization method to calculate the network reliability of the basic idea is according to certain standards will network is decomposed into two sub networks, recursively decomposed sub network and on and on, until the subnet cannot be separated, through iteration to get the network reliability. Specifically, factorization method is decomposition according to link work or failure two cases: if the link contract as normal work link, link the two endpoints of compressed into a point; If the link failure the delete link, the link of two endpoints in the network. And with state enumeration method, the principle of a class method, do not pay the product and the method to calculate the network reliability, using the factorization method to calculate the network reliability needs constantly to deal with the network. The original use factor decomposition method to solve the network reliability is only applicable to the reliable network nodes, but in real life, the nodes in the network may be failure, therefore, need to consider the node failure.

\section{5) State Space Decomposition Method}

State space decomposition method for network reliability by Doulliez, brought forward the basic idea is to put the state space is decomposed into a collection of three states: a collection of acceptable condition, unacceptable state set and unclear status. For the amount of data transferred on the network constraints can be successfully (can't) by source points to lodge, said the network state is acceptable (unacceptable). Each unclear status contains the acceptable and unacceptable state. State space decomposition method to keep $U$ break down into smaller A, N and $\mathrm{U}$, until $U$ finish all decomposition, at this time all the probabilities is the sum of the reliability of the whole network.

State enumeration method, the principle of a class method, don't pay the product and the method, the factorization method, state space decomposition method of the precise algorithm in the study of evaluation of network reliability has important function and meaning, however, due to the network reliability assessment is NP problem, the accurate algorithm more commonly used in small networks or with special topology network reliability. For the large network, the approximate algorithm to calculate the network reliability. 


\section{System Model}

Distributed mechanism of label switching path contains a set of work path and a backup path, the network structure in charge - distributed label switching path, we must consider two functions: total construction cost as the objective function of the network and network reliability as constraints.

1. The network construction cost

Let $R=\left\{r_{1}, r_{2}, \cdots, r_{|k|}\right\}$ said in MPLS network to connect a LER for all disjoint paths, among them, $|R|$ said after the disjoint paths of total number, $r_{k}$ said the article $\mathrm{k}$ disjoint paths. Connect a LER for a LSP unit time needs the amount of data transferred to $\mathrm{D}$, is divided into more and through multiple connection the LER for a child of the LSP to transmit. П Said costs - distributed collection of label switching path problem work path, $|\Pi|$ said path is the total number of work, $w_{k}$ should be assigned $r_{k}$ according to the bandwidth. Because the total bandwidth allocated to each work path should not less than $\mathrm{D}$, we have assigned to each work path is equal to the total bandwidth $\mathrm{D}$, namely

$$
D=\sum_{r_{k} \in \Pi} w_{k}
$$

Build path can produce building costs. Unit assumes that each link bandwidth of the cost of building is the same, a path of building cost is equal to the path assigned to the products of the bandwidth and the path length, among them, the length of the path to the path contains the number of links. Let $\left|r_{k}\right|$ the total number of contained in the link $r_{k}, \mathrm{c}\left(r_{k}\right)$ to represent the cost of building $r_{k}, \mathrm{c}\left(r_{k}\right)$ can be determined by the type:

$$
c\left(r_{k}\right)=w_{k} *\left|r_{k}\right|
$$

$r_{\text {spare }} \in R$ said alternate paths, $C_{\text {working }}(\Pi)$ said all work path construction costs, $C_{\text {sparse }}\left(r_{\text {spare }}\right)$ said standby path construction costs, said the network the total building costs. Is after all work path construction costs and spare road building costs, $C_{d l s p}\left(\Pi, r_{s p a r e}\right)$ is after all work path construction costs and spare road building costs, namely:

$$
C_{d l s p}\left(\Pi, r_{\text {spare }}\right)=C_{\text {working }}(\Pi)+C_{\text {spare }}\left(r_{\text {spare }}\right)
$$

where $C_{\text {working }}(\Pi)$ denote as follows

$$
C_{\text {working }}(\Pi)=\sum_{r_{k} \in \Pi} w_{k} *\left|r_{k}\right|
$$

Due to $r_{\text {spare }}$ the failure of the working path for fault tolerance, the spare bandwidth $w_{\text {spare }}$ should not be less than the bandwidth of any a work path, let $w_{\text {spare }}$ is equal to the largest work path bandwidth, namely:

$$
w_{\text {spare }}=\max _{k \in \Pi} w_{k}
$$

Building size $w_{\text {spare }}$ for the construction of the backup path $r_{\text {spare }}$ of bandwidth costs are as follows: 


$$
C_{d l s p}\left(\Pi, r_{\text {spare }}\right)=\sum_{r_{k} \in \Pi} w_{k} *\left|r_{k}\right|+\max _{k \in \Pi} w_{k} *\left|r_{\text {spare }}\right|
$$

\section{Network Reliability}

Because each path allocated bandwidth is equal to the path to the amount of data transferred, so need to transfer the amount of data that can be sent over the network in a unit time. In cost - distributed label switching path problem, network reliability $\operatorname{Re}\left(\Pi, r_{\text {spare }}\right)$ should not be less than the given network reliability limit $\mathrm{Re}_{T H}$ :

$$
\operatorname{Re}\left(\Pi, r_{s p a r e}\right) \geq \operatorname{Re}_{T H}
$$

$\operatorname{Re}\left(\Pi, r_{\text {spare }}\right)$ can pass to 1 minus the network reliability to obtain. If there is $\operatorname{Re}\left(\Pi, r_{\text {spare }}\right)$ only one path, equals to 1 minus the work and the failure probability of alternate paths. $\alpha_{k}$ said $r_{k}$ can transmit the data on the assigned to the path of success probability $\operatorname{Re}\left(\Pi, r_{\text {spare }}\right)$, and $|\Pi|=1$ can be determined by type (10) :

$$
\operatorname{Re}\left(\Pi, r_{\text {spare }}\right)=1-\left(1-\alpha_{k}\right) *\left(1-\alpha_{\text {sparse }}\right) \quad|\Pi|=1
$$

Among them, the associated $\alpha_{k}$ with $r_{k}$ contained in the link state. In this article, there are two kinds of state: each link work or failure. The failure of different link state is independent of each other. Assume that each link the probability of successful transmission data is the same, $\beta$ said to link the probability of successful transmission data, $\alpha_{k}$ can be determined by type (11):

$$
\alpha_{k}=\beta^{\left|r_{k}\right|}
$$

Based on the type (10) with type (11), when $|\Pi|=1$ the expression of $\operatorname{Re}\left(\Pi, r_{\text {spare }}\right)$ is as follows:

$$
\operatorname{Re}\left(\Pi, r_{\text {spare }}\right)=1-\left(1-\beta^{\left|r_{k}\right|}\right) *\left(1-\beta^{\left|r_{\text {spare }}\right|}\right) \quad|\Pi|=1
$$

Based on this article only USES a backup path, when $|\Pi| \geq 2$, there are two kinds of MPLS network failure events. The first failure event is any a work path and backup path is failure; The second failure events is at least two working path vector effect. Make unrel1 $\left(\Pi, r_{\text {spare }}\right)$ and unrel2 $\left(\Pi, r_{\text {spare }}\right)$ respectively the first and the second kind of the probability of failure events. When $|\Pi| \geq 2, \operatorname{Re}\left(\Pi, r_{\text {spare }}\right)$ can be determined by the type:

$$
\operatorname{Re}\left(\Pi, r_{\text {spare }}\right)=1-\text { unrel } 1\left(\Pi, r_{\text {spare }}\right)-\text { unrel } 2\left(\Pi, r_{\text {spare }}\right), \quad|\Pi| \geq 2
$$

The opposite of at least two working path failure event has two: no job path failure and there is only one path failure events. Therefore, unrel $2\left(\Pi, r_{\text {spare }}\right.$ ) equals to 1 minus the sum of the probability of the two events.

$$
\text { unrel2 }\left(\Pi, r_{\text {spare }}\right)=1-\left(\alpha_{1} * \alpha_{2} * \cdots * \alpha_{|\Pi|}+\sum_{r_{k} \in \Pi} \operatorname{pr}\left(\bar{r}_{k}\right)\right)
$$

Network reliability expression such as type (15): 


$$
\operatorname{Re}\left(\Pi, r_{\text {spare }}\right)=\left\{\begin{aligned}
1-\left(1-\beta^{\left|r_{k}\right|}\right) *\left(1-\beta^{\left|r_{\text {sarer }}\right|}\right) & \text { if }|\Pi|=1 \\
\beta^{\left(\left|r_{1}\right|\left|r_{2}\right|+\cdots+\left|\eta_{\Pi}\right|\right)}+\alpha_{\text {spare }} * \sum_{r_{k} \in \Pi} \operatorname{pr}\left(\overline{r_{k}}\right) & \text { if }|\Pi| \geq 2
\end{aligned}\right.
$$

Cost - distributed label switching path problem research goal is to find the optimal after work and spare under the condition of meet the reliability limit, make the network successful transmission D total construction cost minimum unit of data network. Cost - distributed mathematical model label switching path problem are as follows

$$
\left\{\begin{array}{c}
D=\sum_{r_{k} \in \Pi} w_{k} \\
\operatorname{Re}\left(\Pi, r_{\text {spare }}\right) \geq \mathrm{Re}_{T H}
\end{array}\right.
$$

\section{The Proposed Scheme}

$C_{d l s p}\left(\Pi, r_{\text {spare }}\right)$ with $\operatorname{Re}\left(\Pi, r_{\text {spare }}\right)$ all related to choice of the length of the path, $C_{d l s p}\left(\Pi, r_{\text {spare }}\right)$ and $\operatorname{Re}\left(\Pi, r_{\text {spare }}\right)$ there is a coupling relationship. Let $\mathrm{r}$ all paths rearrange a vector, which is according to the length of the elements in $r$ size ascending order. $\mathrm{R}$ are defined as follows:

$$
r=\left(r_{1}, r_{2}, \cdots, r_{|R|}\right)
$$

Based on the vector $\mathrm{r}$ after the arrangement, $C_{\text {dlsp }}\left(\Pi, r_{\text {spare }}\right)$ and $\operatorname{Re}\left(\Pi, r_{\text {spare }}\right)$ can be decoupled. Therefore, CR - DLSP problem can be divided into two sub-problems: Cost - DLSP and Rdiability - DLSP subproblems. Because of the Cost - DLSP subproblems have been solved by Sohn et al, we will briefly describe Sohn et research Cost - DLSP subproblems to get the main conclusion. Then, for Reliability - DLSP subproblems, we will put forward some conclusions for problem solving, and a Reliability - DLSP algorithm is proposed to meet the conditions of work and the backup path. Finally, the optimal solution of CR - DLSP question by comparing the Cost - DLSP and Reliability - DLSP solutions to subproblems.

\subsection{DLSP Sub Solution of the Problem}

In Cost - DLSP subproblems, regardless of the CR - DLSP problem in network reliability constraints. Cost - DLSP subproblems research goal is to identify successful transmission unit D data volume and the total construction Cost of MPLS network minimum optimal work and alternate paths, and a corresponding bandwidth allocation for each path. Network obtained by derivation of $K, 1<<(|R|-1)$ work path of the total building cost minimum selection criteria, i.e., the article will need to transfer the amount of data the average assigned to work on the path to the transmission, the work and the standby path just take $\mathrm{R} \mathrm{K}+1$ before the element can take $(K+1$ path length of the path of least), and backup path before the $K+1$ are positioned in one of the elements.

$P_{K}$ said any work path set $1 \leq K \leq(|R|-1), \quad C_{d l s p}\left(P_{K}, r_{\text {spare }}\right)$ said work $P_{K}$ and backup path $r_{\text {spare }}$ respectively and build the total network cost, min $C_{d l s p}\left(P_{K}, r_{\text {spare }}\right)$ said the minimum value $C_{d l s p}\left(P_{K}, r_{s p a r e}\right), P_{K+1}^{*}=\left\{r_{1}, \cdots, r_{i}, \cdots, r_{K+1}\right\}$ said before work and alternate paths to take $\mathrm{r} K+1$ the set of elements. Therefore, the Cost - DLSP subproblems 
can be simplified into the work of network total construction Cost minimum path on the number of article $K_{\text {cost }}$.

Let $w_{\text {work }}$ and $w_{\text {spare }}$ respectively and is assigned to the bandwidth of the work and backup path, and can be determined by the type (18):

$$
w_{\text {work }}=w_{\text {spare }}=D / K_{\text {cost }}
$$

Due to Cost - DLSP subproblems without considering the limitation of network reliability, Cost - the best solution of the DLSP subproblems can not ensure its limit satisfy the network reliability. In order to find the optimal working CR - DLSP questions and alternate path, Cost - we need to decide the optimal solution of DLSP subproblems meets the network reliability, and according to the judgment to find the optimal solution of CR - DLSP problem.

Cost - is the best solution of the DLSP subproblems work and backup path in $r$ $K_{\text {cost }}+1$ before, and can be any one of the +1 . Reliability - the solution set of DLSP subproblem is working and the standby path in $\mathrm{r}+1$ before, and take this/C + 1 in the element which has nothing to do. Due to Cost - DLSP and Reliability DLSP subproblems solution are respectively take $\mathrm{r} / \mathrm{Ccost}+1$ before and take the r's former $\mathrm{K}+1,1<\mathrm{K}<K_{R E}$ element, and the arrangement of the elements of $\mathrm{K}+1$ position has nothing to do with +1 are positioned in one of the elements in this matter. This suggests that the Cost - DLSP and Reliability - DLSP subproblem solutions are based on the same search criteria, therefore, CR - DLSP problem of optimal work before and standby path of solution set is the $\mathrm{r}+1$, and are positioned in the $K_{\text {cost }}+1$. Because of the Cost - DLSP subproblems of the optimal solution does not meet the network reliability constraints, we need further to determine the optimal solution of CR - DLSP problem. According to conclusion Sohn et al, for we need to compare/QxjST - 1 and value of the size. The process is the same. Through repeated comparison process, we may safely draw the conclusion: when > KRE, CR - DLSP problem of optimal before work and backup path of solution sets $r+1$ element, and $K_{\text {cost }}+1$ are positioned in one of the elements in this matter.

In determining the CR - after the optimal number of work DLSP problem $|\Pi|=1$, we need to consider each path bandwidth allocation. According to Sohn et. al., to the conclusion that when the network needs to transfer the total amount according to average coordination on the work path, network total construction cost minimum.

\section{Simulation Results}

Article 20 the MPLS network disjoint paths known $|\mathrm{r}|=$ $\{2,3,3,3,3,4,4,4,5,6,6,6,7,8,8,9,9,9,10,10\}, \mathrm{D}=15==0.9$ and 0.975 . And before $\mathrm{r} \mathrm{K}$ +1 are positioned in one of the elements has nothing to do, in this case, we have according to the proposed algorithm, a Kre $==6$. Through Sohti put forward algorithm, can find out $=4$. Figures 1 and 2 are given respectively with $\mathrm{K}(1<\mathrm{K}<$ article 19) work after changing the number of graphs. Figure 1 and 2, respectively, and is proved to trend of change with the increase of $\mathrm{K}$ : namely increases with the increase of K. At that time, decreases with increasing; At that time, increases with increasing.

Because, through the type (18), available $|\Pi|=4$. Then, by type (18), available $w_{C R-D L S P}=D /|\Pi|=15 / 4=3.75$. Therefore, to the problem of two cases of CR - DLSP its optimal work path number is 4 . 


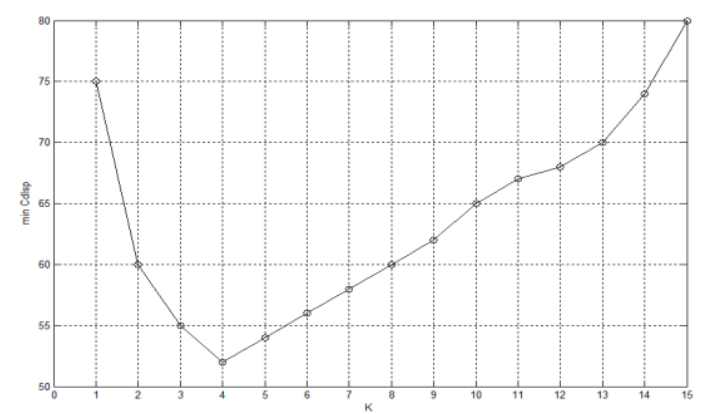

Figure 1. $\min C_{d l s p}\left(P_{K}, r_{\text {spare }}\right)$ with K Work Path Map

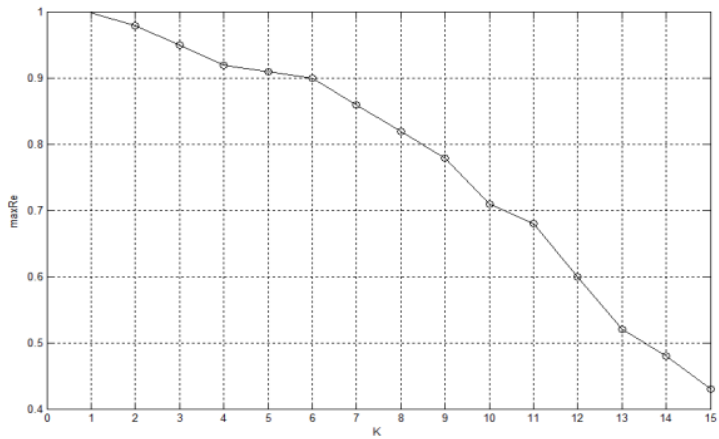

Figure 2. $m a x \operatorname{Re}\left(P_{K}, r_{\text {spare }}\right)$ with K Work Path Map

Namely its optimal collection of work and backup path for, and alternate paths to any one $r_{i}, \mathrm{i}=1,2, \ldots, 5$. Assigned to each path on the bandwidth of 3.75 , the corresponding network total construction cost a minimum of 52.5.

\section{Conclusion}

Work path, this paper analyses the given circumstances, after work and backup path optimization selection and the design of the corresponding bandwidth allocation problem. For multi-protocol label switching network, the network reliability and network is put forward to build cost decoupling method, under meet reliability constraints, solve the network establishment of cost minimization and the standby path optimization selection and bandwidth optimization allocation problem, the original NP problem into a polynomial time can solve the problem.

\section{References}

[1] C. C. Jane, W. H. Shen and Y. W. Laih, "Practical sequential bounds for approximating two-terminal reliability", European Journal of Operational Research, vol. 195, no. 2, (2009), pp. 427-441.

[2] Y. K. Lin, "Spare routing reliability for a stochastic flow network through two minimal paths under budget constraint", IEEE Transactions on reliability, vol. 59, no. 1, (2010), pp. 2-10.

[3] Y. K. Lin and C. T. Yeh, "Multi-objective optimization for stochastic computer networks using nsga-ii and topsis", European Journal of Operational Research, vol. 218, no. 3, (2012), pp. 735-746.

[4] C. J. Colboum, "The combinatorics of network reliability", New York: Oxford University Press, (1987).

[5] W. C. Yeh, "A simple minimal path method for estimating the weighted multi-commodity multistate unreliable networks reliability", Reliability Engineering and System Safety, vol. 93, no. 1, (2008), pp. 125-136.

[6] W. C. Yeh, "An improved sum-of-disjoint-products technique for the symbolic network reliability analysis with known minimal paths", Reliability Engineering and System Safety, vol. 92, (2007), pp. 260-268S. 
[7] M. J. Zuo, Z. Tian and H. Z. Huang, "An efficient method for reliability evaluation of multistage networks given all minimal path vectors", IEEE Transactions on Reliability, vol. 39, no. 8, (2007), pp. 811-817.

[8] Y. Y. K. Lin and C. T. Yeh, "Maximal network reliability with optimal transmission line assignment for stochastic electric power networks via genetic algorithms", Applied Soft Computing, vol. 1, no. 2, (2011), pp. 2714-2724.

[9] Y. K. Lin and L. C. L. Yeng, "Evaluation of network reliability for computer networks with multiple sources", Mathematical Problems in Engineering, (2012).

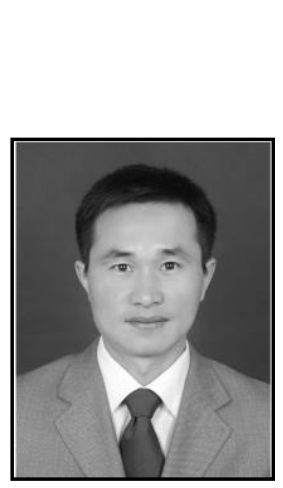

\begin{abstract}
Authors
Yongfeng Cui received the BS degree in Computer Science and Technology from Henan Normal University and the MS degree in Computer Application Technology from Huazhong University of Science and Technology, China in 2000 and 2007 respectively. He is currently researching on Computer Application Technology (CAT).
\end{abstract}

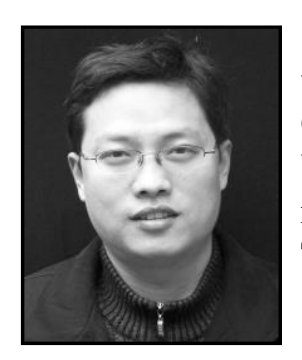

Wei Liu received the BS degree in computer science and technology from Henan Normal University and the MS degree in Computer Technology from The PLA Information Engineering University, China in 1996 and 2006 respectively. He is currently researching on Network Technology (NT) and Computer Application Technology (CAT).

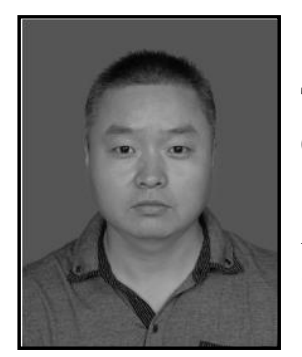

Ya $\mathbf{L i}$ received the BS degree in Computer Science and Technology from Northeast Normal University and the MS degree in Computer Technology from Beijing Jiaotong University, China in 1995 and 2005 respectively. He is currently researching on Computer Application Technology (CAT) and Mobile Internet Technology (MIT). 